\title{
The analysis of distributed control and information processing in adaptive systems: a biologically motivated approach
}

\author{
Roberto R. Kampfner \\ Computer and Information Science, School of Engineering, The University of Michigan-Dearborn, Dearborn, MI 48128 (USA)
}

(Received May 15th, 1991)

(Revision received December 13th, 1991)

\begin{abstract}
Biological systems have evolved hierarchical, distributed control structures that greatly enhance their adaptability. Two important determinants of biological adaptability considered here are: (i) the pattern of distribution of self-control capabilities; (ii) the degree of programmability of information processing. In this paper we model organizations as goal-oriented, adaptive systems, possessing properties similar to those of biological systems. We use the notion of implicit control (defined as the capability of self-control that is embedded in a system's own dynamics) in the analysis of the impact of specific patterns of distribution of control and information processing on the adaptability of organizations. A principle of design of organizational information systems, that captures important aspects of adaptability-preserving strategies of information processing in biological systems, is stated in terms of the implicit control concept.
\end{abstract}

Keywords: Adaptability; Biological information processing; Distributed control; Distributed information processing; Hierarchical systems; Implicit control.

\section{Introduction}

Biological systems have developed fundamental adaptive capabilities through evolution. These capabilities include analog, adaptable modes of information processing, and information processing strategies that preserve or enhance adaptability. An understanding of the mechanisms and strategies underlying these fundamental capsibilities may be instrumental for the successful development of information processing support in complex, adaptive systems.

In this paper we analyze the effect of introducing computerized information processing systems on the adaptability of organizations. The organizational control systems model, or OCSM, allows for the representation of basic structural features of organizational systems (Kampfner, 1987). We use this model for the representation of structural features of organizations that are critical for their adaptability. On the basis of this representation we then apply the adaptability framework (Conrad, 1983) to the analysis of the effect of specific information processing architectures on the adaptability of particular organizational structures. One of the structural features of interest is what we call implicit control (Kampfner, 1989). The degree of implicit control of a system reflects the degree of autonomy of its operational subsystems, that is, the extent to which they control their own behavior. Since any functional subsystem in an organization can be conceived as having some degree of self-control, the implicit control of a system may be distributed in many different ways throughout the organization's hierarchy.

The interplay of structure and dynamics is an essential feature of biological adaptability. The 
tradeoff theorem of information processing (Conrad, 1985), captures fundamental aspects of this interplay. It asserts that an information processing system cannot be in high degree and at the same time programmable, evolvable and efficient. While digital computers are clearly on the programmability side of this tradeoff, biological systems are on the evolvability and efficiency side. It is common experience that programmable systems are not in general amenable to gradual, evolutionary change. For this reason, we associate adaptability with the evolvability and efficiency side of the tradeoff theorem.

For the analysis of the structure-dynamics interplay in organizational systems, I focus on the effect that specific schemes of distribution of control and information processing have on the adaptability of organizations. From the point of view of structure, the aspects considered concern the degree of centralization of control and information processing resources, and the degree of intercommunication between functional units in the organization. From the point of view of the dynamics of control and information processing we consider the degree of programmability of information processing.

We take the pattern of distribution of implicit control as a basis for determining a convenient degree of distribution of information processing throughout the various levels of organizational control and function. The idea is to provide information processing support to an organization in a manner that preserves or enhances its adaptability. To this effect, the adaptability provided by the implicit control available in an organization must be either preserved or compensated when the existing information system is modified, or a new information system is developed. We therefore propose the preservation or compensation of the adaptability provided by the implicit control available in an organization as a basic design objective for organizational information systems. Since this design objective is clearly consistent with the development of information systems that effectively support goal achievement, we present it as a design principle for organizational informa- tion systems. We also analyze certain important tradeoffs involved in the distribution of control and information processing in organizations and outline a methodology for the application of the design principle.

The paper is organized as follows. Section 2 presents a review of the literature relevant to the understanding of strategies and mechanisms of adaptability and information processing in biological systems. The view of organizations as hierarchical, adaptive systems is presented in Section 3, where we describe the OCSM as a tool for the representation of hierarchical control structures in goal-oriented organizations. The concept of implicit control in hierarchical systems is also explained in this section, with reference to the OCSM. In Section 4 we introduce Conrad's framework of adaptability and apply it to the analysis of hierarchical control structures. The design principle for organizational information systems is presented in Section 5 , as a basis for the allocation of information processing support between control units in organizations. A methodology for the application of the design principle is also presented from an information systems development perspective.

\section{Control and information processing in adaptive systems}

Let us review some key contributions to the understanding of the principles and mechanisms underlying adaptability in biological systems. The proposal of cybernetics (Wiener, 1961) is perhaps the best known attempt to systematize the study of communication and control mechanisms in biological systems with the view to their implementation in artificial systems. Early in the development of cybernetics, Ashby's principle of requisite variety (Ashby, 1956) established fundamental requirements for the complexity of the control function of systems dealing with uncertain environments. In the broad context of systems theory, Laszlo (1972) defined wholeness and order, adaptive self-stabilization, adaptive self-organization, and systemic hierarchy as properties essential for 
the description of the behavior of natural systems. Another important development is Rosen's proposal of self-repair and replication as fundamental capabilities of living systems. Rosen formalized their role in modeling biological behavior in his metabolism-repair systems (Rosen, 1972). More recently, Casti (1989) formalized these capabilities in the context of linear systems and outlined extensions to this formulation, dealing with the more complicated cases of networks, nonlinearities and evolution.

The study of information-processing strategies used by biological systems must seriously consider the relationship between structure and dynamics and its role in biological adaptation. In this respect, Conrad has explored the role of macromolecular structures in evolutionary gradualism (Conrad, 1979), and in mediating analog modes of information processing (Conrad, 1974, 1976). The role of structure in shaping dynamics in biological systems has been discussed by Kampfner and Conrad (1983) in the context of evolutionary learning systems. The unique ability of biological systems to develop an adaptability-enhancing relationship between their structural and dynamical features has also been investigated at the level of the physical foundations. At this level, Conrad has developed the skipping model (Conrad, 1989), that explains the ability of biological systems to unmask a fundamental irreversibility of the time evolution caused by disturbing equilibrium relations in the vacuum structure.

Another approach to modeling the behavior of complex organizational systems facing uncertain environments is the theory of hierarchical, multilevel systems (Mesarovic, 1970). Mesarovic models a multilevel organizational hierarchy as a family of interacting subsystems, some of which are decision-making units arranged hierarchically. These decision-making units, however, are not considered as control units within functional subsystems, but as problemsolving levels within a decision-making process. Mesarovic's framework focuses on the formulation of mathematical models of hierarchical, multilevel systems. It considers different types of hierarchical structures, and discusses their application to specific kinds of organizations. However, it does not address the structuredynamics issue. Therefore, it does not consider the requirements that an organization's goal structure imposes on the structure and dynamics of their information processing and control systems.

The viable system model, or VSM (Beer, 1984), is another conceptual model of hierarchical, complex systems. The VSM sees organizations as recursive structures with five main interactive subsystems in each viable component (or subsystem). The VSM addresses basic structural and dynamical needs of viable systems. The VSM, however, does not consider an explicit role of information processing in control, or the effect of alternative forms of information processing on the success of the control function.

We should mention here that organization theorists have also acknowledged uncertainty as a major determinant of the need to process information. Galbraith (1974), for example, argues that the greater the task uncertainty, the greater the amount of information that must be processed among decision-makers in order to achieve a given level of performance.

An important framework for the analysis of strategies and mechanisms used by biological systems is provided by the theory of adaptability (Conrad, 1983). This framework, that characterizes the role of variability in biological adaptability in statistical terms, provides the conceptual foundations that we need for the analysis of information processing strategies in hierarchical systems.

\section{A hierarchical model of organizational control}

The organizational control systems model, or OCSM (Kampfner, 1987), provides a hierarchical representation of the structure of an organization and its control system. It describes the relationship between control and operational units at all levels and in all the functional areas of the organization. An important assumption of 
the OCSM is that the goals of an organization can be decomposed into a hierarchy of subgoals, and that there is an adaptive, functional subsystem responsible for the achievement of each of these subgoals. The hierarchical nature of organizations is essential for their adaptability, since it allows for the coordinated interaction of functional subsystems, each responsible for the achievement of a specific subgoal. The hierarchy concept implies that each functional subsystem is itself adaptive. In the face of a relatively uncertain environment it must have a control subsystem monitoring and coordinating its activities in order to achieve the particular set of organizational subgoals for which it is responsible. Since each functional subsystem is responsible for a unique set of organizational goals, the OCSM identifies with each functional subsystem a unique control subsystem responsible for the achievement of such goals. Because of the oneto-one correspondence between goals and functional subsystems, the goal hierarchy imposes an isomorphic hierarchy on the set of functional subsystems. Since each functional subsystem must have a unique control subsystem, there is also a corresponding hierarchy on the set of control subsystems.

As mentioned above, the interplay of structure and dynamics is vital for the adaptability of biological systems. In this respect, our main focus is on the demands and limitations that the structural features of a system impose on its behavior from the point of view of adaptability. Given the hierarchical control structure of a particular organization, our goal is to identify and characterize the patterns of distribution of information processing resources that fulfil its information needs, but which do not produce a negative effect on the adaptability of the organization as a whole. An important characteristic of these patterns of distribution of information processing is the degree of programmability of information processing at each level of the hierarchy. In this respect, the OCSM allows for the explicit representation of all the relevant levels of control of each area of function in a given organization. As described below, this representation facilitates the analysis of the impact of introducing specific information processing structures on adaptability.

Formally, we define three binary relations on the set of functional subsystems of an organization as follows. Let $O_{\mathrm{S}}=\left\{S_{i, j}\right\}$ be the set of functional subsystems, where $i$ represents a level in the organization's functional hierarchy, and $j$ identifies the $j$ th subsystem at that level. The SUBSYSTEM relation, is defined as the set SUBSYSTEM $=\left\{\left\langle S_{i, j}, S_{i}+1, k\right\rangle: S_{i, j}\right.$ is a subsystem of $S_{i+1, k}$, that is, the goal of $S_{i, j}$ is a subgoal of $S_{i}+1, k$ ). We write SUBSYSTEM$\left(S_{i, j}, S_{i+1, k}\right)$ to mean that $\left\langle S_{i, j}, S_{i+1, k}\right\rangle \epsilon$ SUBSYSTEM.

Let $S_{0} \subseteq O_{\mathrm{S}}$, be the set of control subsystems in an organization. Another useful relation is the relation CONTROLS, defined between a control subsystem $S_{i, j_{0}}, S_{i, j_{0}} \in S_{0}$, and the operational subsystems $S_{i, j_{k}}$, within the same system, at the same level. This relation is defined as the set CONTROLS $=1<S_{i, j_{0}}, S_{i, j_{k}}>: S_{i, j_{0}}$ assigns to $S_{i, j_{k}}$ a subgoal of their parent system, and monitors its performance toward the achievement of this goal \}. For all pairs $<S_{i, j_{0}}, S_{i, j_{k}}>\epsilon$ CONTROLS, we require that both SUB$\operatorname{SYSTEM}\left(S_{i, j_{0}}, S_{i}+1, m\right)$ and $\operatorname{SUBSYSTEM}\left(S_{i, j_{k}}\right.$, $\left.S_{i+1, m}\right)$ hold. We also write $\operatorname{CONTROLS}\left(S_{i, j_{0}}\right.$, $S_{i, j_{k}}$ ) to mean that $\left\langle S_{i, j_{0}}, S_{i, j_{k}}>\in\right.$ CONTROLS.

We also define the relation REPORTS-TO on the set of control subsystems of an organization as the set REPORTS-TO $=\left\{<S_{i, j_{0}}, S_{i}+1, k_{0}\right\rangle$ : $S_{i, j_{0}}$ is the internal control of $S_{i}+1, m$ and $S_{i+1, k_{0}}$ is its controller, that is, both SUB$\operatorname{SYSTEM}\left(S_{i, j_{0}}, S_{i}+1, m\right)$ and CONTROLS$\left(S_{i+1, k_{0},}, S_{i}+1, m\right)$ hold \}. Here, $S_{i, j_{0}}, S_{i}+1, k_{0} \epsilon$ $S_{0}$. We also write REPORTS-TO $\left(S_{i, j_{0}}, S_{i}+1, k_{0}\right)$ to mean that $S_{i, j_{0}}$ reports to $S_{i}+1, k_{0}$, a higherlevel control subsystem.

We refer to the ordering imposed on a set of functional subsystems by the SUBSYSTEM relation, a partial ordering, as the functional hierarchy, and to the one defined over the control subsystems by the REPORTS-TO relation as the control hierarchy.

Relations SUBSYSTEM, CONTROLS and REPORTS-TO uniquely specify the structure of an organization for the purposes of the analysis of the distribution of control and information 


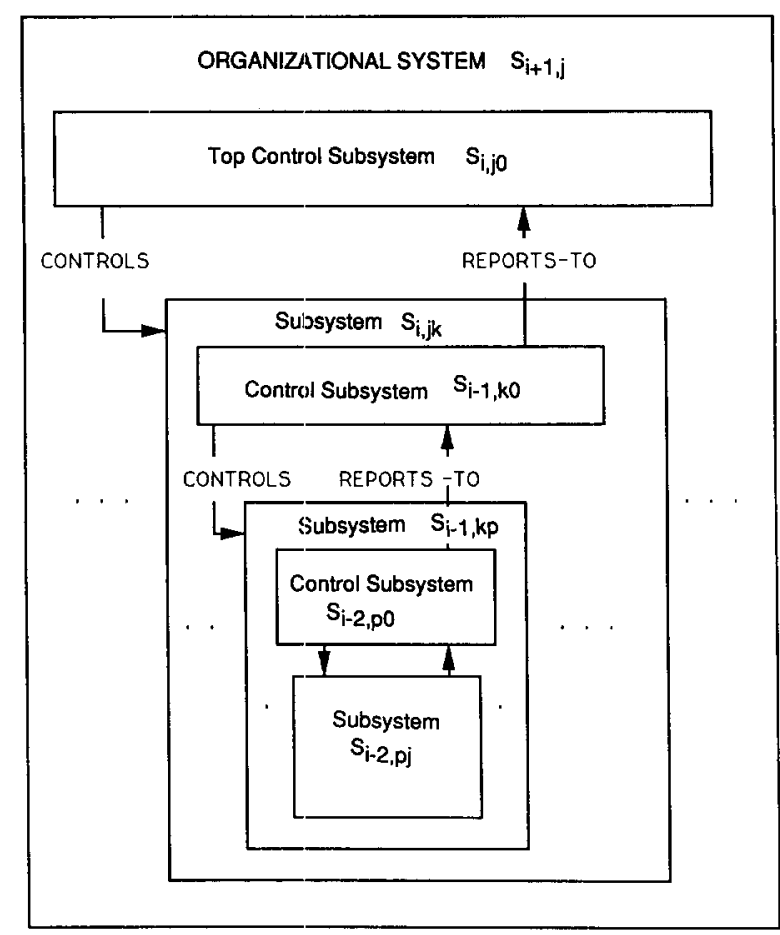

Fig. 1. Schematic view of the functional and control hierarchies as identified with the OCSM. Arrows joining pairs in the REPORTS-TO and the CONTROLS relations are identified in the figure. Subsystems are shown enclosed within the boundaries of their parent systems. Each subsystem has only one control subsystem represented by a wide rectangle. Tall, narrower rectangles represent operational subsystems.

processing. Figure 1 describes schematically the relationship between the functional and control hierarchies of an organization. In particular, it shows the relationship between the SUBSYSTEM, CONTROLS, and REPORTS-TO relations.

In the OCSM, any functional subsystem must have only one control subsystem, its internal control. For instance, $S_{i, j_{k}}$ (see Fig. 1) is subdivided into $S_{i-1, k_{0}}, \ldots, S_{i-1, k_{p}}, \ldots, S_{i-1, k_{m}}$. However, the only control subsystem of $S_{i, j_{k}}$ is $S_{i-1, k_{0}}$. This means that for any $S_{i-1, k_{0}} \in S_{0}$, $S_{i-1, k_{p}}, S_{i, j_{k}} \in O_{\mathrm{S}}$
$\operatorname{SUBSYSTEM}\left(S_{i-1, k_{0}}, S_{i, j_{k}}\right)$

and

$\operatorname{SUBSYSTEM}\left(S_{i-1, k_{p}}, S_{i, j_{k}}\right)$

implies

$\operatorname{CONTROLS}\left(S_{i-1, k_{0}}, S_{i-1, k_{p}}\right)$

Briefly, if $S_{i-1, k_{0}}$ is a control subsystem, and $S_{i-1, k_{p}}$ is an operational one that belongs to the same parent system, $S_{i, j_{k}}$ say, then $S_{i-1, k_{0}}$ controls $S_{i-1, k_{p}}$ since the former is the unique internal control of $S_{i, j}$, their parent system.

Also, for any $S_{i-1, k_{0}}, S_{i, j_{0}} \in S_{0}$ and $S_{i-1, k_{p}}$, $S_{i, j_{k}} \in O_{\mathrm{S}}$

$\operatorname{CONTROLS}\left(S_{\left.i-1, k_{0}, S_{i-1, k_{p}}\right)}\right.$

and

$\operatorname{SUBSYSTEM}\left(S_{i-1, k_{0}}, S_{i, j_{k}}\right)$

and

$\operatorname{CONTROLS}\left(S_{i, j_{0}}, S_{i, j_{k}}\right)$

implies

REPORTS-TO $\left(S_{i-1, k_{0}}, S_{i, j_{0}}\right)$

This means that the internal control of a system reports to that system's controller.

This property of the OCSM imposes on the subsystems it models the constraint that, for the purposes of explicit control, the communication between control subsystems must be vertical, and made through the appropriate channels. This means that the explicit control activities are centralized within each functional subsystem's explicit control. This property makes no assumption about the actual physical location of the control subsystem (or unit) of a system. It requires, however, that all the control activities of a system be coordinated by its control subsystem. 
An important parameter of the structure of an adaptive system is the scope of its control subsystem, or internal control (Kampfner, 1987). Informally, the scope of an (internal) control subsystem is the extent to which it monitors the behavior of its parent system. The scope of a control subsystem is thus determined by the nature and magnitude of the goals it is responsible for, and by the constraints under which these goals must be achieved. Thus, the greater the constraints that a system's control subsystem has, for the purposes of furthering the goals of its parent system, the smaller its scope is.

We also refer to the control subsystem of a system as its explicit control. According to the OCSM, the explicit control of a system is that subsystem responsible for coordinating the behavior of the other (operational) subsystems in an explicit manner. It involves the use of models and symbolic information processing. The implicit control of a system, on the other hand, is identified with the self-regulating capabilities of its operational subsystems. As such it is responsible for those sub-goals that are not directly monitored by its (explicit) control subsystem. More precisely, we define the implicit control of a system as the capability of selfcontrol of its functional subsystems.

In biological systems, the self-control capabilities of specific functional subsystems are embedded into their own dynamics. Some levels of metabolic functions, for example, are regulated by the action of protein enzymes that determine the reaction rates associated with various biochemical pathways. As another example, at the elementary level of brain function, the dynamics of the functional subsystems responsible for the transmission of nerve impulses involve the action of specific neurotransmitters, such as acetylcholine and norepinephrine. The regulation of the activity of these transmitters is clearly mediated and, to a great extent, performed as an integral part of the physiology of brain processes. In organizational systems, the use of symbolic information processing, that involves the manipulation of systems that represent objects in some domain, exists at several levels in a functional or control hierarchy. A low- level function, such as that of determining the date at which a customer order will be fulfilled, usually involves the use of models and symbolic information processing. A common feature between the customer order processing organizational function and the metabolism- and nerve impulse transmission-related biological functions is that they both possess some degree of self-control. A basic difference between them, however, is that while the first one is supported by a typically programmable mode of information processing, the others use an analog, nonprogrammable mode. Although, according to our definition, they both form part of the implicit control of their respective parent systems, as discussed in the next section, they differ in their potential contribution to adaptability. We consider lower-level subsystems in a hierarchical system as an integral part of the dynamics of higher level ones.

The degree of self-control of a system is closely related to the scope of its control subsystem. If the scope of a control subsystem is large, then its implicit control is correspondingly small. The OCSM provides basic types of objects and relations for the representation of the control and functional hierarchies that embody the structural features that define the relationship between implicit and explicit control in particular organizations. The scope of control of the various subsystems of a given organization can also be evaluated on the basis of the OCSM representation. Consequently, the pattern of distribution of self-control capabilities that exists in a given organization can be represented in the OCSM in a natural way. Corresponding dynamical features of an organization's control system, can be analyzed in terms of the degree of programmability of information processing. An adaptability based measure of the degree of implicit control existing within an organization is developed in the next section.

\section{Hierarchical control and the adaptability framework}

In this section, we introduce some basic notions of adaptability theory (Conrad, 1983) and 
use the notion of hierarchical control defined by the OCSM to develop a framework for the analysis of the impact of specific patterns of distribution of control and information processing on the adaptability of hierarchical, adaptive systems.

Adaptability requirements for a system $S$ can be expressed using the following, fundamental inequality (Conrad, 1983):

$$
U(\bar{S})-U(\bar{S} \mid \bar{E})+U(\bar{E} \mid \bar{S}) \geq U(\bar{E})
$$

where $U(\bar{S})$ is the potential uncertainty of the transition scheme of system $S, U(\bar{E})$ is the potential uncertainty of the transition scheme of its environment, $U(\bar{S} \mid \bar{E})$ is the potential uncertainty of the transition scheme of the system given the state transition of the environment, and $U(\bar{E} \mid \bar{S})$ is the potential uncertainty of the transition scheme of the environment given the state transition of the system. Given in terms of the degree of decorrelation between the behavior of the environment and the behavior of the system, $U(\bar{E} \mid \bar{S})$ represents the maximum indifference with respect to its environment that the system can have without losing its ability to maintain its characteristic pattern of activity.

The difference $U(\bar{S})-U(\bar{S} \mid \bar{E})$ represents the information that the behavior of the environment provides about the contemporaneous behavior of the system. This difference is related to the anticipation of the system as follows: $U(\bar{S} \mid \bar{E})$ rejresents the lack of ability of the system to anticipate the state of the environment. It grows as the correlation of the behavior of the system with that of the environment decreases. Since $U(\bar{S})$ represents the behavioral uncertainty of the system, the difference $U(\bar{S})$ $U(\bar{S} \mid \bar{E})$ can be interpreted as the reduction in the uncertainty of the behavior of the system that occurs once the corresponding state transition of the environment is given. According to Eqn. (3), an increase in $U(\bar{S})-U(\bar{S} \mid \bar{E})$ results in an increase in the adaptability of system $S$.

Basically, this difference can be increased in two ways:

(1) by increasing $U(\bar{S})$, the potential variability of the system;
(2) by reducing $U(\bar{S} \mid \bar{E})$, its potential inability to anticipate the behavior of the environment.

In the case of (1), the increase in $U(\bar{S})$ must be sufficiently correlated with the behavior of the environment, otherwise it would not contribute to the adaptability of $S$. In the case of (2), the reduction in $U(\bar{S} \mid \bar{E})$ is achieved through an increase in the correlation of the behavior of the system with the behavior of the environment.

When the increase in adaptability of a system $S$ corresponds entirely to an increase in the adaptability of its operational subsystems, we say that it corresponds to an increase in its implicit control. The implicit control of a system may be implemented as an integral part of the dynamics of the system being controlled. In goal-oriented, hierarchical systems, (explicit) control subsystems with varying scopes of control are defined at various hierarchical levels. The explicit control of an operational subsystem, however, forms part of its self-control capabilities. As such, it is part of the implicit control of its parent system. Therefore, the increase in the implicit control of a system may result in an increase in the explicit control at lower levels.

The increase in the explicit control of a system $S$ may result in a loss of adaptability caused by an increase in the effective use of information processing, especially when it is accompanied by an increase in the independence of its operational subsystems. Obviously, for the adaptability of a system to be increased, or at least maintained, there must be a compensation for any loss of adaptability caused by the increase in the effective use of information processing originated in its explicit control. An increase in the correlation of the behavior of the system with that of the environment, that originates in the explicit control of a system, and which corresponds to a decrease in $U(\bar{S} \mid \bar{E})$, must be achieved in such a way that either the difference $U(\bar{S})-U(\bar{S} \mid \bar{E})$ does not decrease in value, or any decrease is compensated by a greater degree of indifference with respect to the environment.

Let us now analyze in more detail, the impact of introducing an increase in the effective use of information processing on the adaptability of a 
system. Here, by effective use of information processing we mean the use of information by the explicit control of a system. In organizational systems, the use of models for planning and control, and hence the use of computerbased information systems, is generally correlated with the effective use of information processing. According to adaptability theory (Conrad, 1983), an increase in the effective use of information processing in a system causes an increase in the predictivity of its transition scheme. This increase, especially if accompanied by an increase in the independence of the system, may result in a loss of adaptability. In the remainder of this section, we formalize the concept of distribution of implicit and explicit control in a system's hierarchy from the standpoint of adaptability.

Let us denote by $A_{\mathrm{S}}$ the adaptability of system $S$, and denote by $A^{\prime}{ }_{S}$ its adaptability after some increase in the use of explicit control, that is,

$$
A_{\mathrm{S}}=U(\bar{S})-U(\bar{S} \mid \bar{E})+U(\bar{E} \mid \bar{S})
$$

and

$$
A_{\mathrm{S}}^{\prime}=U\left(\bar{S}^{\prime}\right)-U\left(\bar{S}^{\prime} \mid \bar{E}\right)+U\left(\bar{E} \mid \bar{S}^{\prime}\right)
$$

For the system to be sufficiently adaptable we must have $A_{\mathrm{S}} \geq U(\bar{E})$ before more explicit control is added and $A^{\prime}{ }_{\mathrm{s}} \geq U(\bar{E})$ afterwards.

The change in adaptability caused by an increase in a system's explicit control, let us call it $A_{\mathrm{C}}$, is given by

$$
A_{\mathrm{C}}=A_{\mathrm{S}}^{\prime}-A_{\mathrm{S}}
$$

Using Eqns. (4) and (5), we have

$$
\begin{aligned}
A_{\mathrm{C}}= & U\left(\bar{S}^{\prime}\right)-U\left(\bar{S}^{\prime} \mid \bar{E}\right)+U(\bar{S} \mid \bar{E})-U(\bar{S}) \\
& +U\left(\bar{E} \mid \bar{S}^{\prime}\right)-U(\bar{E} \mid \bar{S})
\end{aligned}
$$

We need $A_{\mathrm{C}} \geq 0$ in order to preserve adaptability. This results in

$$
U\left(\bar{S}^{\prime}\right)-U\left(\bar{S}^{\prime} \mid \bar{E}\right)+U(\bar{S} \mid \bar{E})-U(\bar{S})
$$

$$
+U\left(\bar{E} \mid \bar{S}^{\prime}\right)-U(\bar{E} \mid \bar{S}) \geq 0
$$

Rearranging terms, we have

$$
\begin{aligned}
& U(\bar{S} \mid \bar{E})-U\left(\bar{S}^{\prime} \mid \bar{E}\right) \geq U(\bar{E} \mid \bar{S})-U\left(\bar{E} \mid \bar{S}^{\prime}\right) \\
& \quad+U(\bar{S})-U\left(\bar{S}^{\prime}\right)
\end{aligned}
$$

This means that any combined decrease in the potential variability of the system, and in its indifference with respect to the behavior of the environment that the system can afford, must be matched by an increase in the correlation of its behavior with that of the environment.

An increase in the explicit control of a system is necessarily accompanied by an increase in the effective use of information processing that is involved in the use of models for planning and control. According to adaptability theory, the effective use of information processing results in an increase in the predictivity of the transition scheme of the system (Conrad, 1983). The increase in predictivity in the transition scheme of the system corresponds to a decrease in its potential uncertainty, that is, it implies that $U(\bar{S})>U\left(\bar{S}^{\prime}\right)$. This condition, in turn, reflects a loss of adaptability of the system, unless its behavior is sufficiently correlated with the behavior of the environment. When a loss of adaptability occurs, it has to be compensated in order for the system to maintain its ability to achieve its goals.

Assuming that the indifference that the system can tolerate with respect to the environment does not change with the increase of explicit control, that is, that $U\left(\bar{E} \mid \bar{S}^{\prime}\right)=U(\bar{E} \mid \bar{S})$, we have

$U(\bar{S} \mid \bar{E})-U\left(\bar{S}^{\prime} \mid \bar{E}\right) \geq U(\bar{S})-U\left(\bar{S}^{\prime}\right)$

According to Eqn. (10), the increase in anticipation must be equal to or greater than the loss of behavioral uncertainty of the system.

Let us now consider the ways in which this loss of adaptability can be compensated in a hierarchical adaptive system.

In general, the compensation for lost adaptability can be provided to the system in any of three basic ways: 
(i) by another system, external to $S$;

(ii) by an increase in the system's explicit control;

(iii) by an increase in its implicit control.

In the case of (i), where the needed adaptability is provided by an external system, the com. pensation is provided as an increase in the indifference component of the system's adaptability. In the case of (ii), an increase in the explicit control of the system requires a further increase in the effective use of information processing. This, in turn, requires an increase in the availability of information about the behavior of the environment, as well as the ability to process that information effectively. In the case of (iii), the increase in implicit control requires an increase in the behavioral uncertainty of the system, in a manner as correlated as possible with the behavior of the environment. This increase in the behavioral uncertainty of the system must take place within the boundaries of one or more of its operational subsystems. As such, it entails an increase in the self-control capabilities of these subsystems. Case (iii) illustrates those situations in which reinforcing the delegation of control responsibilities to lower levels in the organization's hierarchy, and the distribution of information processing for the support of these, more decentralized control structures, is the design option chosen. Here is where the advantages of a hierarchical organization for adaptability become apparent. But adaptability can be provided in many different ways with the use of implicit control.

The concept of the partial state of a system (Conrad, 1983) is useful in this context. The partial state of a system is a description of its state given in terms of variables that describe basic features of the behavior of its lower-level subsystems. A description of the complete state of the system would include more detailed aspects of the behavior of such subsystems. The partial state description of a system thus eliminates the need of referring to unnecessary details concerning the behavior of lower-level subsystems. We use the partial state concept to apply the OCSM description to the analysis of the adaptability of specific control structures. In particular, we define the partial state of a system in terms of the variables incorporated in the models used by its (explicit) control subsystem.

Let us consider a simplified hierarchical system $S$, with only two functional subsystems, $S_{1}$ and $S_{2}$. The behavioral uncertainty of $S$ can be expressed in terms of the uncertainty of its partial scheme, $\dot{S}_{0}$ and the complete schemes of its subsystems $\bar{S}_{1}$ and $\bar{S}_{2}$ that is,

$$
U(\bar{S})=U\left(\dot{S}_{0} \bar{S}_{1} \bar{S}_{2}\right)
$$

Another useful concept is that of effective entropy. This concept (Conrad, 1983) identifies additive components of the behavior of a hierarchical system. Each of these components represents the uncertainty that originates in the interaction of specific subsets of the set of subsystems. The concept of effective entropy is particularly useful here because of its additive character. Let us denote by $U_{\mathrm{e}}\left(\dot{S}_{0}\right), U_{\mathrm{e}}\left(\bar{S}_{1}\right)$, and $U_{\mathrm{e}}\left(\bar{S}_{2}\right)$ the effective entropies of the partial scheme of $S$, and of the complete scheme of its subsystems $S_{1}$ and $S_{2}$, respectively. The behavioral uncertainty of $S$ can then be expressed as

$$
U\left(\dot{S}_{0} \bar{S}_{1} \bar{S}_{2}\right)=U_{\mathrm{e}}\left(\dot{S}_{0}\right)+U_{\mathrm{e}}\left(\bar{S}_{1}\right)+U_{\mathrm{e}}\left(\bar{S}_{2}\right)
$$

According to Eqn. (11) we, therefore, have,

$$
U(\bar{S})=U_{\mathrm{e}}\left(\dot{S}_{0}\right)+U_{\mathrm{e}}\left(\bar{S}_{1}\right)+U_{\mathrm{e}}\left(\bar{S}_{2}\right)
$$

Similarly, for $U\left(\bar{S}^{\prime}\right)$ we have, in terms of effective entropies,

$$
U\left(\bar{S}^{\prime}\right)=U_{\mathrm{e}}\left(\dot{S}_{0}{ }^{\prime}\right)+U_{\mathrm{e}}\left(\bar{S}^{\prime}{ }_{1}\right)+U_{\mathrm{e}}\left(\bar{S}_{2}{ }_{2}\right)
$$

The conditional entropies can also be expressed as effective entropies. For example, the entropy of the original transition scheme, given the state transition of the environment, can be expressed as

$U(\bar{S} \mid \bar{E})=U_{\mathrm{e}}\left(\dot{S}_{0} \mid \bar{E}\right)+U_{\mathrm{e}}\left(\bar{S}_{1} \mid \bar{E}\right)+U_{\mathrm{e}}\left(\bar{S}_{2} \mid \bar{E}\right)$

Similarly, the conditional entropy of the transition scheme of $S$, after the increase in the effective use of information processing has taken place, can be expressed as 


$$
\begin{aligned}
U\left(\bar{S}^{\prime} \mid \bar{E}\right)= & U_{\mathrm{e}}\left(\dot{S}_{0}^{\prime} \mid \bar{E}^{\prime}\right)+U_{\mathrm{e}}\left(\bar{S}_{1}^{\prime} \mid \bar{E}\right) \\
& +U_{\mathrm{e}}\left(\bar{S}_{2}^{\prime} \mid \overline{E^{\prime}}\right)
\end{aligned}
$$

The conditional entropy of the transition scheme of the environment given the state transition of a system, represents the indifference that the system can show with respect to that environment without losing its ability to function. These entropies, before and after the increase in the explicit control of the system has taken place, are given by Eqns. (17) and (18), respectively.

$$
\begin{aligned}
U(\bar{E} \mid \bar{S})= & U_{\mathrm{e}}\left(\bar{E} \mid \dot{S}_{0}\right)+U_{\mathrm{e}}\left(\bar{E} \mid \bar{S}_{1}\right)+U_{\mathrm{e}}\left(\overline{\bar{E}} \mid \bar{S}_{2}\right) \\
U\left(\bar{E} \mid \bar{S}^{\prime}\right)= & U_{\mathrm{e}}\left(\bar{E} \mid \dot{S}_{0}^{\prime}\right)+U_{\mathrm{e}}\left(\bar{E} \mid \bar{S}_{1}^{\prime}\right) \\
& +U_{\mathrm{e}}\left(\bar{E} \mid \bar{S}_{2}^{\prime}\right)
\end{aligned}
$$

In a hierarchical system, each subsystem, with the exception of those at the lowest level, can be further divided into lower-level subsystems. Whenever a subsystem admits decomposition into lower-level subsystems, we can express its transition scheme in terms of the product of its partial scheme with the complete schemes of its subsystems at the next lower level (cf. Conrad, 1983).

Let us assume, for example, that subsystem $S_{1}$ is subdivided into subsystems $S_{11}$ and $S_{12}$ with $S_{10}$ as its explicit control. In analogy with Eqn. (11), the transition scheme of $S_{1}$ could be expressed as

$$
\bar{S}_{1}=\dot{S}_{10} \bar{S}_{11} \bar{S}_{12}
$$

The behavioral uncertainty of $S_{1}$ can thus be given in terms of the uncertainty of this product, that is,

$$
U\left(\bar{S}_{1}\right)=U\left(\dot{S}_{10} \bar{S}_{11} \bar{S}_{12}\right)
$$

In terms of the effective entropies, we can write

$U\left(\bar{S}_{1}\right)=U_{\mathrm{e}}\left(\dot{S}_{10}\right)+U_{\mathrm{e}}\left(\bar{S}_{11}\right)+U_{\mathrm{e}}\left(\bar{S}_{12}\right)$ where the effective entropies are determined as before.

According to the OCSM framework, the partial state of an organizational system, or any of its subsystems, is given in terms of the variables that the control subsystem, its explicit control, uses in order to perform its function. These variables must describe the state of the system so that it can be adequately monitored by its control subsystem. The goals and parameters assigned by a control subsystem to the operational subsystems it controls, and the feedback subsequently returned by these subsystems, must therefore be given in terms of these variables.

Thus, in an organizational system $S$, the partial scheme $\dot{S}_{0}$ describes its transition scheme from the point of view of its explicit control at the top level, say top management. Similarly, each operational subsystem of the organization has its control subsystem, or explicit control, that views such an operational subsystem in terms of its partial scheme. This identification of the partial scheme of a system with the way its explicit control views it provides a useful connection between the OCSM and the theory of adaptability. In particular, it allows us to analyze key tradeoffs involved in the distribution of control and information processing.

More precisely, since we have defined the partial state of a system $S$ in terms of the variables used by its control subsystem to monitor its behavior, the uncertainty of the partial scheme is exactly the uncertainty of the control subsystem. Moreover, as indicated above, the complete scheme of a system can be given in terms of the product of its partial scheme and the complete schemes of its subsystems (cf. Eqns. (11) and (20). Consequently, as shown in Eqns. (13) and (21), the behavioral uncertainty of a system $S$ can be expressed as the sum of the effective entropy of its partial scheme, and the effective entropies of the complete transition schemes of its subsystems. The anticipation term of the adaptability of $S$ can also be given in terms of effective entropies, as shown by Eqns. (15) and (16). This allows us to express the relative contributions of the implicit control and the explicit control of $S$, to its adaptability. 
Let us denote by $A_{\mathrm{S}}$ the total contribution of the anticipation component of adaptability. According to Eqn. (3), the fundamental inequality, this contribution is given by

$$
A_{\mathrm{S}}=U(\bar{S})-U(\bar{S} \mid \bar{E})
$$

The individual contributions to adaptability of the anticipation components of the control subsystem $\dot{S}_{0}$ and of subsystems $S_{1}$ and $S_{2}$ of $S$, are defined as follows. The contribution of the control subsystem is given by

$$
A_{0}=U_{\mathrm{e}}\left(\dot{S}_{0}\right)-U_{\mathrm{e}}\left(\dot{S}_{0}^{\prime} \mid \bar{E}\right)
$$

Similarly, the contribution of $S_{1}$ is

$$
A_{1}=U_{\mathrm{e}}\left(\dot{S}_{1}\right)-U_{\mathrm{e}}\left(\dot{S}_{1} \mid \overline{E^{\prime}}\right)
$$

Finally, the contribution of $S_{2}$ is

$$
A_{2}=U_{\mathrm{e}}\left(\dot{S}_{2}\right)-U_{\mathrm{e}}\left(\dot{S}_{2} \mid \bar{E}\right)
$$

The contributions $A_{1}$ and $A_{2}$ originate in the implicit control, since $A_{1}$ and $A_{2}$ are both operational subsystems of $S$. $A_{0}$ originates in the control subsystem and, therefore, represents the contribution of the explicit control of $S$.

Now we can define the relative contributions of the implicit and the explicit control of $S$ to its adaptability. The total contribution of the anticipation component to the adaptability of $S$, can also be expressed as

$A_{\mathrm{S}}=A_{0}+A_{1}+A_{2}$

Let us denote by $\alpha_{\mathrm{E}}$ the relative contribution of the explicit control to the anticipation of $S$, and by $\alpha_{\mathrm{I}}$ the relative contribution of its implicit control. These are expressed as

$\alpha_{\mathrm{E}}=A_{0} /\left(A_{0}+A_{1}+A_{2}\right)$

for the explicit control, and

$\alpha_{\mathrm{I}}=\left(A_{1}+A_{2}\right) /\left(A_{0}+A_{1}+A_{2}\right)$

for the implicit one.

A measure of the degree of centraliza- tion/decentralization of control in a hierarchical, adaptive system, is given by the expression

$\Psi_{\mathrm{C}}=\sum_{i=1}^{n} w_{i} \alpha_{\mathrm{E} i}$

where $\alpha_{\mathrm{E} i}$ is the relative contribution of the explicit control to the anticipation of subsystem $S_{i} ; W=\left\{w_{i}\right\}$ is a set of relative weights, such that,

$$
\sum_{i=1}^{n} w_{i}=1
$$

and

$w_{j} \leq w_{k}$, for $j<k$

Here, $0 \leq i, j, k \leq n$, where $n$ is the number of control subsystems in the hierarchy.

Condition (ii) imposes a partial order on $W$. The SUBSYSTEM relation also imposes a partial order on $\left\{S_{i}\right\}$, the set of subsystems. To fulfil its role, the ordering in $W$ must preserve the ordering imposed on the set $\left\{S_{i}\right\}$ by the SUBSYSTEM relation. This means that the following additional condition is required. For $i, k \in I$, and control subsystems $S_{i}, S_{k}$,

$$
i<k \text { iff }\left\langle S_{i}, S_{k}\right\rangle \in \text { SUBSYSTEM }
$$

Within this ordering, particular weight assignments can be given to the various control subsystems that emphasize the impact of the centralization/decentralization of specific aspects of control.

$\Psi_{C}$ is a weighted average of the distribution of the relative contribution to anticipation of each of its control subsystems. Although a measure of the degree of centralization of control in an organization, as defined by $\Psi_{C}$ is difficult to obtain in any precise manner, the framework developed here certainly helps to evaluate the effect of computerized information processing on adaptability in a manner that is consistent with the functional meaning of information processing. A basic methodology for the analysis of this effect in the context of information systems development is presented in the next section. 


\section{A design principle for organizational infor- mation systems}

When a computer-based information system is developed and installed in an organization, it influences the organization's decision-making structure, especially the degree of centralization of decision-making and the programmability of information processing that supports the control function. This influence has a direct impact on the adaptability of the organization and, consequently, has to be evaluated properly before major design decisions are made.

As mentioned above, the hierarchy of goals determines an organization's structure in terms of its functional and control hierarchies. The anticipation required by the various functional subsystems can be provided in various combinations of implicit and explicit control. An important observation here is that the information processing support provided to the explicit control at some level in the organization's hierarchy can be seen as implicit control from the standpoint of a higher-level subsystem.

Because of its effect on the programmability of information processing, the impact of information processing provided for the support of the explicit control on adaptability must be always carefully evaluated. A framework for this evaluation is provided by a design principle for organizational information systems (Kampfner, 1989) which advocates for the adequate use of the implicit control available in an organization's (hierarchical) control structure. The design principle states that in order to provide effective information processing support to an organization's control system, the use of the available implicit control must be attempted first. Then, the support to the explicit control must be expanded, if necessary. In any case, provisions must be made for the compensation of any loss of adaptability caused by the increase in the use of models for planning and control.

The design principle guides the allocation of information processing support to both the implicit and the explicit components of organizational control. By focusing on the adequate balance between implicit and explicit control, the design principle provides a solid basis for the development of information processing system architectures that effectively support an organization's control function. More precisely, it helps find a scheme of distribution of information processing that matches the degree of centralization/decentralization of the organization's control structure.

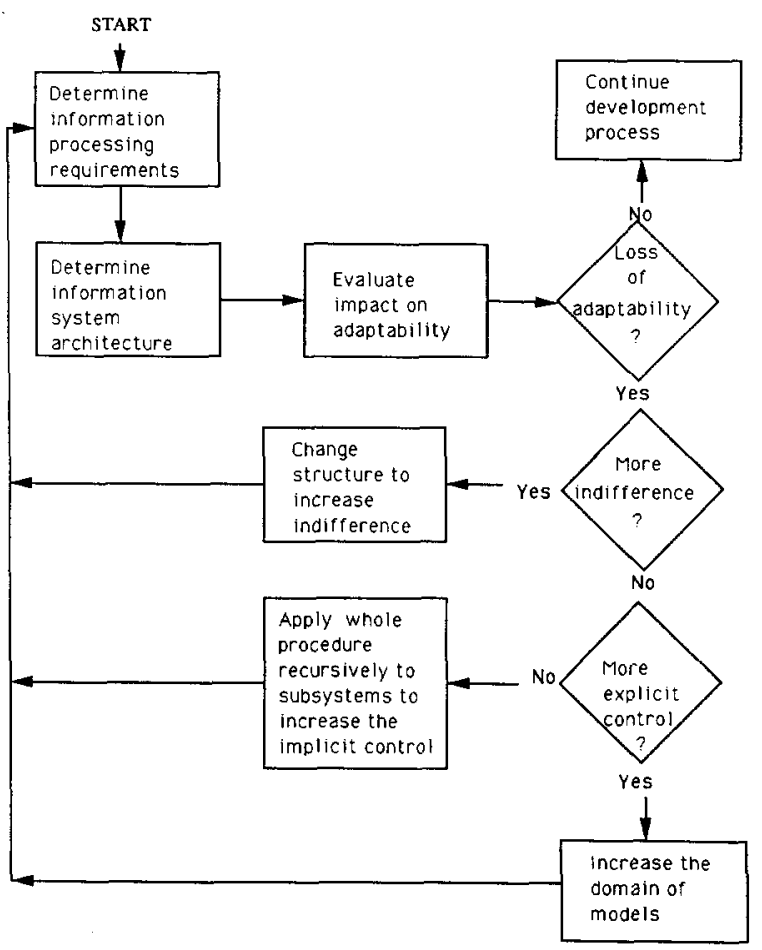

Fig. 2. Flowchart of the process followed in the analysis of the impact of introducing new automated information processing functions on the adaptability of an organization. The process is iterative. After the structure of the control system is determined, or adjusted, its information processing requirements are determined. These information requirements are used as a basis for determining the architecture of the information system (at the logical level). Each alternative architecture is evaluated for its impact on adaptability. If there is no loss of adaptability, the software development process can continue. Otherwise, the loss of adaptability has to be compensated in any of three possible ways as indicated in the figure. When the implicit control is to be increased, the whole process is applied recursively to each operational subsystem. For each operational subsystem submited to this analysis, including the original system, the cycle is repeated each time new information requirements are determined. 
A basic methodology for the application of the design principle is as follows. The process of evaluating the effect of a proposed increase in automated information processing on the adaptability of the organization, illustrated graphically in Fig. 2, starts with the determination of information processing requirements as the basis for the development of the computer-based information system. The goal is to find an information system architecture capable of satisfying the information needs of the organization. According to the design principle, this means that the requirements of the information system must be satisfied without loss of adaptability. The adaptability criterion is fundamental, since it implies that the organizational functions requiring information are supported in a manner that ensures that the ability of the organization to achieve its goals persists indefinitely.

Once a set of information processing requirements has been determined, their impact on the adaptability of the system can be evaluated. According to adaptability theory, the basic concern is the increase in the predictivity of the transition scheme of the system, especially when such an increase is coupled with a corresponding increase in its independence with respect to related systems.

If there is no loss of adaptability, the development of the software system can proceed, following the proposed scheme for the distribution of control and its associated information processing support. However, if a loss of adaptability associated with the development of the new information processing system is found, it has to be compensated for. This can be done in any of the following ways:

(a) Increasing the indifference of the system. An increase in the indifference component of the adaptability of the system might be possible through structural changes applied to certain subsystems, external to the one receiving the information processing support. If this option is found feasible, then the structural changes required must be carried out, and the corresponding information system requirements must be determined.

(b) Increasing the anticipation provided by the explicit control. The adaptability of a system can also be increased by increasing the power of the computational models incorporated in the information system. The increase in power of the computational models can be achieved by increasing the number of conditions that they consider. This may require a greater degree of interaction of these models with the organizational environment, both internal and external. If this option is found feasible, the augmented models should be considered for implementation, and the corresponding information system requirements determined.

(c) Increasing the implicit control. This option entails the use of control mechanisms that are, together with the associated information processing, embedded into the dynamics of the system. Since the implicit control of a system resides in the behavior of its operational subsystems, the focus of the analysis shifts to the next lower level in the hierarchy, where these systems are located. The analysis of the effect on adaptability in each of these subsystems can be carried out applying this procedure recursively to each of the operational subsystems.

Of course, the information system project should be abandoned if no increase in automated information processing without loss of adaptability is possible, or if it fails to meet conventional criteria for feasibility.

The design of information systems has of course been considered in organization theory as an important aspect of organizational design. Structure, in the form of rules, programs and hierarchy, and dynamic problem solving and communications have been considered important aspects of organizational design (see Gerloff (1985), for example). An information system leading to a convenient type of coordination must, of course, include the right mix of these ingredients. Tushman and Nadler (1978) rate alternative information processing mechanisms, such as the use of rules and programs, hierarchy, planning and goals, vertical information systems, and lateral relations, according to their cost and complexity, and the information capacity of the organization. They argue that information processing capacity 
should meet information processing demand, but that the type of organization, mechanistic or organic, must also be taken into account. Although the need to match information demand and capacity, and to consider the type of organization has been acknowledged, criteria for information systems design that effectively take into account the latter have not been defined, at least to this author's knowledge. On the other hand, the design principle stated here provides a useful criterion for the determination of the appropriate architecture of the information processing system that will support a given organizational control structure. In particular, the design principle helps determine the pattern of distribution of information processing that would effectively support a given degree of centralization of control. More specific aspects of the design of the information system such as the degree of distribution of databases, the types of computer networks, modes of processing, and the details of the user interface can, subsequently, be determined on this basis.

\section{Conclusions}

The application of the adaptability framework to the analysis of the distribution of control and information processing in hierarchical systems has important implications. The main one is that there exists a delicate balance between the adaptability of a system and the extent to which it depends on programmable information processing for the support of its control function. This implication acquires a very specific meaning in the context of goal-oriented organizations. The allocation of anticipation to the explicit control of a system, which entails an increase in automated information processing support, generally implies a loss of adaptability that has to be compensated for if the system is to cope effectively with environmental uncertainty.

Secondly, once the use of automated information processing has been increased, if there is a loss of adaptability due to the use of automated information processing, the compensation for the adaptability lost can be made in three dif- ferent ways: (1) by increasing the indifference of the system with respect to its environment; (2) by increasing the system's explicit control; (3) by incorporating self-control capabilities in the behavior of the system, that is, by increasing its implicit control. A convenient choice has therefore to be made between any combination of the three options mentioned above, in order to compensate for the loss of adaptability caused by an increase in the use of information processing. In fact, information system and organization designers must look for a convenient mix of the three options in order to achieve specified objectives, while preserving, or enhancing, the adaptability of the system.

The results reported here indicate that information processing considerations are crucial to effective organizational design, especially from the standpoint of adaptability. They also indicate that the design of an information system must be based on the structure of the control system that it is intended to support. The value of the design principle is that of providing a fundamental criterion for determining the basic architecture of the information processing system. The principle also illustrates how our understanding of information processing strategies used by biological systems can provide a useful basis for the design of information systems that effectively support goal achievement in organizations. Future research on the identification of parameters for the evaluation of tradeoffs between information processing structures for specific types of organizations would subsequently pave the way toward the development of information systems that effectively support organizational function.

\section{References}

Ashby, W.R., 1956, An Introduction to Cybernetics (Wiley, New York).

Beer, S., 1984, The viable system model: its provenance development, methodology, and pathology. J. Oper. Res. Soc. 35, 7-25.

Casti, J.L., 1989, Newton, Aristotle, and the modeling of living systems, in: Newton to Aristotle, Toward a Theory of Models for Living Systems, J.L. Casti and S. Karlqvist (eds.) (Birkhauser, Boston). 
Conrad, M., 1974, Evolutionary learning circuits. J. Theor. Biol. 46, 167- 188 .

Conrad, M., Complementary models of learning and memory. BioSystems 8, 119-138.

Conrad, M., 1979, Bootstrapping on the adaptive landscape. BioSystems 11, 167-180.

Conrad, M., 1983, Adaptability (Plenum Press, New York).

Conrad, M., 1985, On design principles for a molecular computer. Commun. ACM 28, 464-480.

Conrad, M., 1989, Force, measurement, and life, in: Newton to Aristotle, Toward a Theory of Models for Living Systems, J.L. Casti and S. Karlqvist (eds.) (Birkhauser, Boston).

Galbraith, J.R., 1974, Organization design: an information processing review. Interfaces 4, 28-36.

Gerloff, E., 1985, Organizational Theory and Design (McGraw-Hill, New York).

Kampfner, R., 1987, A hierarchical model of organizational control for the analysis of information system requirements. Inform. Systems 12, 243-254.
Kampfner, R., 1989, Biological information processing: the use of information for the support of function. BioSystems 22, 223-230.

Kampfner, R. and Conrad, M., 1983, The role of structure in evolutionary learning, in: Proc. Conf. on Artificial Intellgence, Rochester, MI.

Laszlo, E., 1972, Introduction to Systems Phylosophy (Harper and Row, New York).

Mesarovic, M., Macko, D. and Takahara, Y., 1970, Theory of Hierarchical, Multilevel Systems (Academic Press, New York).

Rosen, R., 1972, Some relational cell models: the metabolism-repair systems, in: Foundations of Mathematical Biology, R. Rosen (ed.) Vol. 2 (Academic Press, New York).

Tushman, M.L. and Nadler, D.A., 1978, Information processing as an integrating concept in organizational design. Acad. Management Rev. 3, 613-624. 